\title{
Bone Marrow, Rib
}

National Cancer Institute

\section{Source}

National Cancer Institute. Bone Marrow, Rib. NCI Thesaurus. Code C77688.

Bone marrow in the rib. 